\title{
HALL EFFECTS ON MHD FLOW PAST AN ACCELERATED PLATE WITH HEAT TRANSFER
}

\author{
J.K. SUNDARNATH \\ Department of Mechanical Engineering \\ Sri Venkateswara College of Engineering \\ Irungattukottai 602 117, Sriperumbudur Taluk, INDIA \\ R. MUTHUCUMARSWAMY* \\ Department of Applied Mathematics \\ Sri Venkateswara College of Engineering \\ Irungattukottai 602 117, Sriperumbudur Taluk, INDIA \\ E-mail: msamy@svce.ac.in
}

\begin{abstract}
Hall current and rotation on an MHD flow past an accelerated horizontal plate relative to a rotating fluid, in the presence of heat transfer has been analyzed. The effects of the Hall parameter, Hartmann number, rotation parameter (non-dimensional angular velocity), Grashof's number and Prandtl number on axial and transverse velocity profiles are presented graphically. It is found that with the increase in the Hartmann number, the axial and transverse velocity components increase in a direction opposite to that of obtained by increasing the Hall parameter and rotation parameter. Also, when $\Omega=M^{2} m /\left(1+m^{2}\right)$, it is observed that the transverse velocity component vanishes and axial velocity attains a maximum value.
\end{abstract}

Key words: Hall parameter, Hartmann number, heat transfer, Grashof's number, Prandtl number.

\section{Introduction}

A magnetohydrodynamic flow refers to the flow of electrically conducting fluids, such as plasmas or ionised gases under a magnetic field. The effect of the magnetic field on the flow of the fluid can be derived using Reynolds's transport theorem and Maxwell's equations. Rossow (1960) first analyzed the Rayleigh problem in MHD without taking into account the induced magnetic field. The flow of electrically conducting fluids in the presence of the magnetic field induces a potential in a direction normal to both the electric and magnetic fields. This phenomenon is known as the Hall effect. The effect of Hall current on the MHD flow of fluids has been analyzed by several authors. Pop (1971) studied the effect of Hall current on an hydromagnetic flow, which is short lived. Maleque and Sattar (2005) investigated the steady laminar MHD flow on a porous rotating disc taking the Hall effect into account. Haytham et al. (2012) studied the magnetohydrodynamic version of the classical Rayleigh problem with Hall effect.

The simultaneous effect of Hall current and rotation on an MHD flow has received significant attention from researchers recently. This is due to the development of improved magnetohydrodynamic generators and magnetohydrodynamic accelerators, lubrication control of high speed spinning machine components under magnetic field, and the study of planetary fluid dynamics. The study of the MHD flow in a rotating environment includes the effect of Coriolis forces, thermal convection current and Hall current. Deka (2008) studied the simultaneous effect of Hall current and rotation on the MHD flow past an

\footnotetext{
* To whom correspondence should be addressed
} 
accelerated plate relative to the rotating fluid. Barik et al. (2013) studied the effect of Hall current on the unsteady MHD flow between two rotating discs with non-coincident parallel axes.

Furthermore the effect of heat transfer in MHD flows is encountered in many cosmological and geophysical phenomena. The sun is a complex MHD system that encompasses several non intuitive phenomena that are not fully understood. For example, the sunspots are regions of reduced temperature on the photosphere of the sun that are short lived. These are generated due to the convection of hot gases caused by the intense magnetic field of the sun (1957). The study of solar dynamics requires the firm understanding of the effect of Hall current on the MHD flow with heat transfer. Bharali and Borkakati (1982) studied the effect of Hall current on the MHD flow with heat transfer characteristics between two porous plates. Ghosh et al. (2009) studied the effects of Hall current on the MHD flow with heat transfer in a parallel plate channel system, with perfectly conducting walls in a rotating frame of reference. Singh (1984) studied the effect of Hall current on hydromagnetic free-convection flow past an accelerated vertical porous plate. Chauhan and Rastogi (2012) investigated the heat transfer effects on the MHD Couette flow in a channel partially filled by a porous medium in the presence of an inclined magnetic field.

In this paper, we study and investigate the simultaneous effects of Hall current and rotation on the MHD flow past an accelerated horizontal plate relative to a rotating fluid, with heat transfer characteristics. A very strong magnetic field is imposed so as to make the Hall effects important. We study the effect of the Hall parameter $\mathrm{m}$, Hartmann number $\mathrm{M}$ and the rotation parameter $\Omega$ (rotating speed with which the plate and the fluid rotate in unison), the Grashof's number Gr and Prandtl number Pr on the axial velocity (along the direction of the plate) and transverse velocity (transverse to the main flow) of the fluid.

\section{Formulation of the problem and solution:}

We consider the unsteady flow of an electrically conducting fluid past an infinitely long flat plate which is electrically non-conducting. The $x$-axis is assumed in the direction of motion of the plate and the $y$ axis and $z$ axis are fixed accordingly in perpendicular directions. The flat plate is supposed to be in the plane $z=0$. Initially the fluid and the plate rotate synchronously with uniform angular velocity $\Omega$. The fluid is then set into motion with uniform acceleration along the $x$-axis. A uniform magnetic field $H_{0}$, is applied in a direction parallel to the $x$-axis. Because of the horizontal homogeneity, the flow parameters depend only on $z$ and the time variable $t^{\prime}$. The initial temperature of the plate and the fluid is assumed to be $T_{\infty}$. After a time $t^{\prime}>0$, the temperature of the plate increases to $T_{w}$, and is regarded to be constant. Also the pressure is assumed to be uniform in the flow. Let $u, v, w$ be the components of the velocity vector $q$.

By the equation of continuity, we have $\nabla \cdot q=0$. This implies that $w=0$ at all places. By the solenoid equation for the magnetic field $\nabla \cdot H=0$, we get $H_{z}=H_{0}=$ constant at all places in the flow. The equation of conservation of electric charges $\nabla \cdot J=0$, gives $J_{z}=0$, as the plate is non-conducting. Also, the fluid that is far away from the plate is assumed to be undisturbed. From all these assumptions, in a rotating frame of reference and using modifications of Ohm's law, the momentum equations for the unsteady flow with heat transfer are given by Ghosh et al. (2009)

$$
\begin{aligned}
& \frac{\partial u}{\partial t^{\prime}}=v \frac{\partial^{2} u}{\partial z^{2}}+2 \Omega_{z} v-\frac{\sigma \mu_{e}^{2} H_{0}^{2}}{\rho\left(1+m^{2}\right)}(u+m v)+g \beta\left(T-T_{\infty}\right), \\
& \frac{\partial v}{\partial t^{\prime}}=v \frac{\partial^{2} u}{\partial z^{2}}-2 \Omega_{z} u+\frac{\sigma \mu_{e}^{2} H_{0}^{2}}{\rho\left(1+m^{2}\right)}(m u-v),
\end{aligned}
$$




$$
\rho c_{p} \frac{\partial T}{\delta t^{\prime}}-\frac{\partial^{2} T}{\partial y^{2}}
$$

Here the second term on the right hand side of Eqs (2.1) and (2.2) is due to small Coriolis force. The boundary conditions are given by

$$
\begin{aligned}
& u=0, \quad T=T_{\infty}, \quad v=0 \quad \text { at } \quad t^{\prime} \leq 0 \text { for all } z \\
& \left.\begin{array}{lllll}
u=A t^{\prime}, & T=T_{w}, \quad v=0 & \text { at } & z=0 \\
u \rightarrow 0, & T \rightarrow 0, \quad v \rightarrow 0 & \text { as } & z \rightarrow \infty
\end{array}\right\} t^{\prime}>0
\end{aligned}
$$

where $A(>0)$ is a constant.

The dimensionless quantities are introduced as follows

$$
\begin{aligned}
& U=\frac{u}{(A v)^{1 / 3}}, \quad V=\frac{v}{(A v)^{1 / 3}}, \quad Z=z(A / \mathrm{v})^{1 / 3}, \\
& t=t^{\prime}\left(A^{2} / \mathrm{v}\right)^{1 / 3}, \quad \Omega=\Omega_{z}\left(v / A^{2}\right)^{1 / 3}, \quad M^{2}=\frac{\sigma \mu_{e}^{2} H_{0}^{2} \mathrm{v}^{1 / 3}}{2 \rho A^{2 / 3}}, \\
& \mathrm{Gr}=\frac{v g \beta\left(T_{w}-T_{\infty}\right)}{\left(A t^{\prime}\right)^{3}}, \quad A=\frac{\left(T-T_{\infty}\right)}{T_{w}-T_{\infty}}, \quad \operatorname{Pr}=\frac{\mu c_{p}}{k} .
\end{aligned}
$$

Together with Eqs (2.1), (2.2) and (2.3) boundary conditions (2.4)-(2.5), and using Eqs (2.6)-(2.8) we have

$$
\begin{aligned}
& \frac{\partial U}{\partial t}=\frac{\partial^{2} U}{\partial Z^{2}}+2 V\left(\Omega-\frac{M^{2} m}{\left(1+m^{2}\right)}\right)-\frac{2 M^{2} U}{\left(1+m^{2}\right)}, \\
& \frac{\partial V}{\partial t}=\frac{\partial^{2} V}{\partial Z^{2}}-2 U\left(\Omega-\frac{M^{2} m}{\left(1+m^{2}\right)}\right)-\frac{2 M^{2} V}{\left(1+m^{2}\right)}, \\
& \frac{\partial \theta}{\delta t}=\frac{1}{\operatorname{Pr}} \frac{\partial^{2} \theta}{\partial Z^{2}} .
\end{aligned}
$$

The initial and final boundary conditions are as follows

$$
U=0, \quad \theta=0, \quad V=0 \quad \text { at } \quad t \leq 0 \text { for all } Z,
$$




$$
\left.\begin{array}{c}
U=t, \quad \theta=1, \quad V=0 \quad \text { at } \quad Z=0 \\
U \rightarrow 0, \quad \theta \rightarrow 0, \quad V \rightarrow 0 \quad \text { as } \quad Z \rightarrow \infty
\end{array}\right\} t>0
$$

Now Eqs (2.9), (2.10) and (2.11) and boundary conditions Eqs (2.12) and (2.13) can be combined to give

$$
\begin{aligned}
& \frac{\partial F}{\partial t}=\frac{\partial^{2} F}{\partial Z^{2}}-2 V\left\{\frac{M^{2} m}{\left(1+m^{2}\right)}+i\left(\Omega-\frac{2 M^{2} m}{\left(1+m^{2}\right)}\right)\right\}+\operatorname{Gr} \theta, \\
& \frac{\partial F}{\partial t}=\frac{\partial^{2} F}{\partial Z^{2}}-F a+\operatorname{Gr} \theta
\end{aligned}
$$

where

$$
\begin{aligned}
& a=\frac{2 M^{2} m}{\left(1+m^{2}\right)}+2 i\left(\Omega-\frac{2 M^{2} m}{\left(1+m^{2}\right)}\right) \quad \text { and } \quad F=U+i V \\
& F=0, \quad \theta=0, \quad \text { at } \quad t \leq 0 \quad \text { for all } \quad Z \text {, } \\
& \left.\begin{array}{l}
F=t, \quad \theta=1, \quad \text { at } \quad Z=0 \\
F \rightarrow 0, \quad \theta \rightarrow 0, \quad \text { as } \quad Z \rightarrow \infty
\end{array}\right\} t>0 .
\end{aligned}
$$

Taking the Laplace transform of Eq.(2.11) and applying boundary condition (2.17) and (2.18), we get

$$
\bar{\theta}=\frac{1}{s} \exp (-Z \sqrt{\operatorname{Pr} s})
$$

Taking the inverse Laplace transform of Eq.(2.19) we get

$$
\theta=\operatorname{erfc}\left(\frac{Z}{2} \sqrt{\frac{\operatorname{Pr}}{t}}\right)
$$

From Eqs (2.15) and (2.21) we get

$$
\frac{\partial F}{\partial t}=\frac{\partial^{2} F}{\partial Z^{2}}-F a+\operatorname{Gr} \operatorname{erfc}\left(\frac{Z}{2} \sqrt{\frac{\operatorname{Pr}}{t}}\right)
$$

Taking the Laplace transform of Eq.(2.21) and by applying boundary conditions (2.17)-(2.18), we get

$$
\bar{F}=\left(\frac{1}{s^{2}}+\frac{\mathrm{Gr}}{a}\left(\frac{1}{s-b}-\frac{1}{s}\right)\right) \exp (-Z \sqrt{s+a})-\frac{\mathrm{Gr}}{a}\left(\frac{1}{s-b}-\frac{1}{s}\right) \exp (-Z \sqrt{\operatorname{Pr} s})
$$

where

$$
b=\frac{a}{\operatorname{Pr}-1}
$$


Taking inverse Laplace transform of Eq.(2.22), we get

$$
\begin{aligned}
& F=\left(\frac{t}{2}-\frac{Z}{4 \sqrt{a}}-\frac{\mathrm{Gr}}{2 a}\right) \exp (-Z \sqrt{a}) \operatorname{erfc}\left(\frac{Z}{2 \sqrt{t}}-\sqrt{a t}\right)+\left(\frac{t}{2}+\frac{Z}{4 \sqrt{a}}-\frac{\mathrm{Gr}}{2 a}\right) \exp (Z \sqrt{a}) \operatorname{erfc}\left(\frac{Z}{2 \sqrt{t}}+\sqrt{a t}\right)+ \\
& \frac{\mathrm{Gr}}{a} \exp \left(\frac{b t}{2}\right)\left(\exp (-Z \sqrt{a+b}) \operatorname{erfc}\left(\frac{Z}{2 \sqrt{t}}-\sqrt{(a+b) t}\right)+\exp (Z \sqrt{a+b}) \operatorname{erfc}\left(\frac{Z}{2 \sqrt{t}}+\sqrt{(a+b) t}\right)\right)+ \\
& -\frac{\mathrm{Gr}}{a} \exp \left(\frac{b t}{2}\right)\left(\exp (-Z \sqrt{b}) \operatorname{erfc}\left(\frac{Z}{2 \sqrt{t}}-\sqrt{b t}\right)+\exp (Z \sqrt{b}) \operatorname{erfc}\left(\frac{Z}{2 \sqrt{t}}+\sqrt{b t}\right)\right)-\frac{\mathrm{Gr}}{a} \operatorname{erfc}\left(\frac{z}{2} \sqrt{\frac{\operatorname{Pr}}{t}}\right) .
\end{aligned}
$$

\section{Results and discussion}

The effects of various parameters on the transient axial velocity and transverse velocity are presented in the following graphs. Figures 1 and 2 show the variation of the axial velocity and transverse velocity with the rotation parameter $\Omega$, for the Grashof number $\mathrm{Gr}=5$, Prandtl number $\operatorname{Pr}=3$, Hall parameter $m=0.5$, Hartmann number $\mathrm{M}=0.5$ and time $T=2$. The negative sign indicates that this component is transverse to the main flow direction in clockwise sense. It is observed that the transient axial velocity and transverse velocity increases with the increase in the rotation parameter $\Omega$. The negatice sign for the transverse velocity component shows that this component is transverse to the main flow in the clockwise direction. Also, when $\Omega=M^{2} m /\left(1+m^{2}\right)$, the transverse velocity component vanishes and axial velocity attains the maximum value, which is the case in the absence of heat transfer also (Barali and Borkakati, 1982).

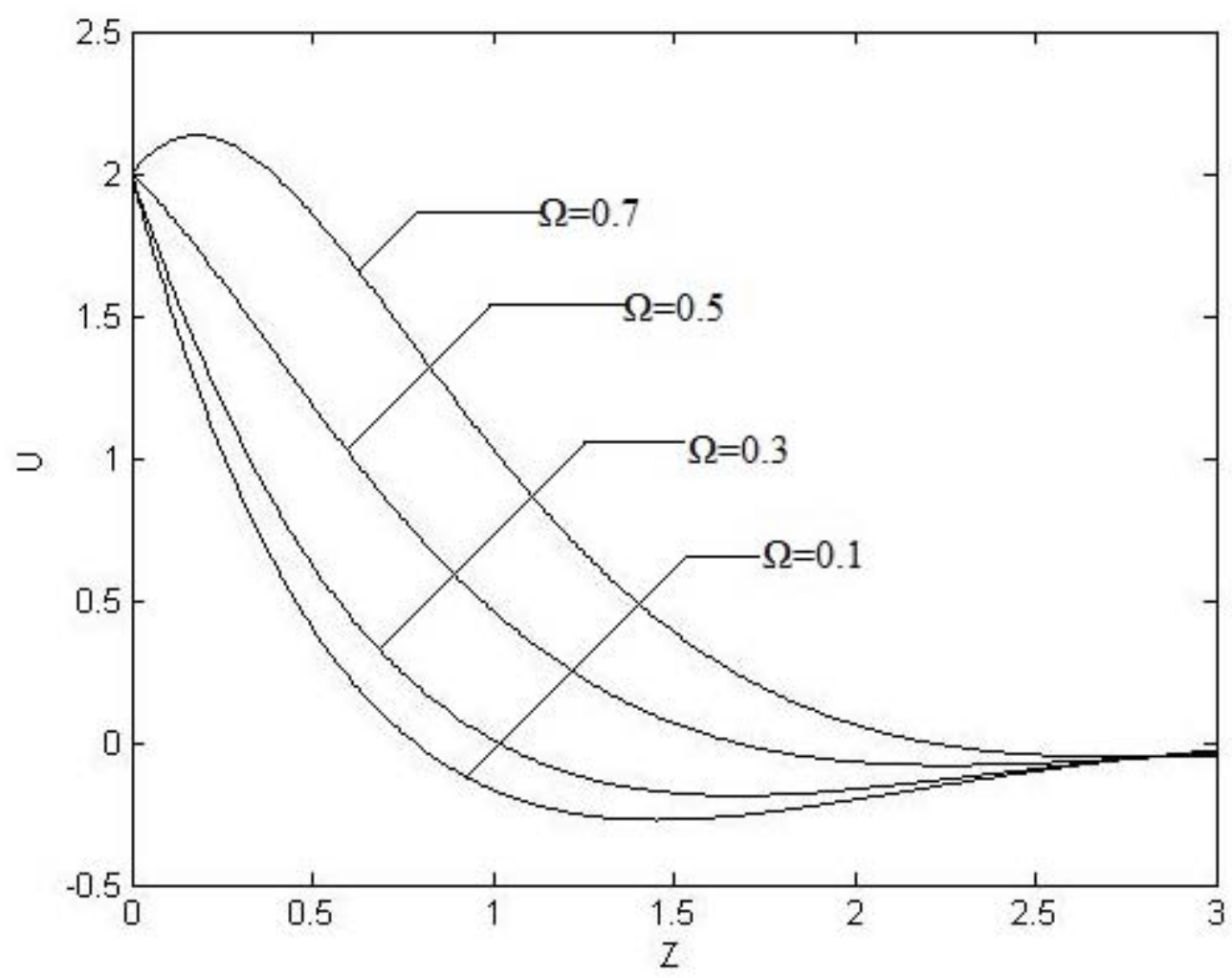

Fig.1. Axial velocity profile for $\mathrm{M}=0.5, \mathrm{~m}=0.5, \mathrm{Gr}=5, \operatorname{Pr}=2$ and $T=2$. 


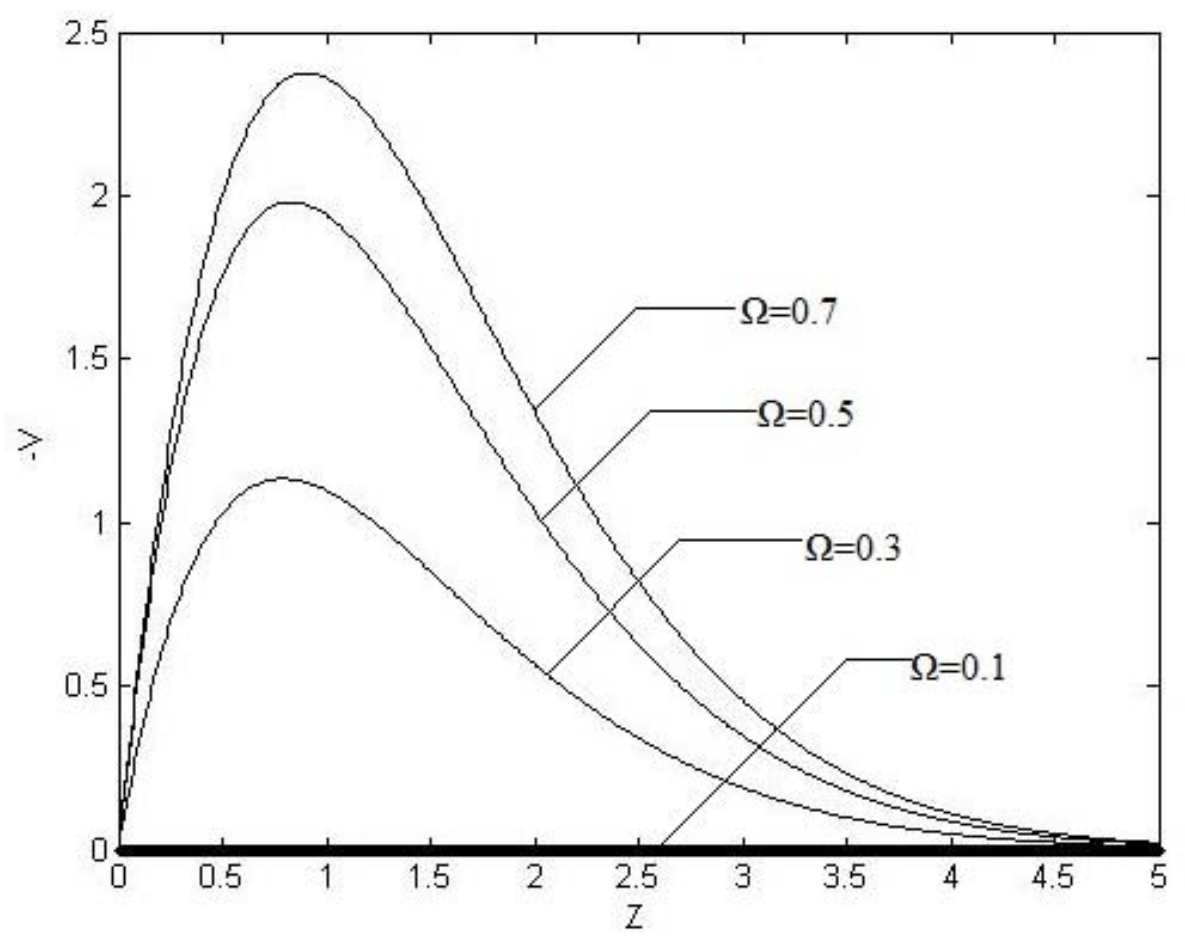

Fig.2. Transverse velocity profile for $\mathrm{M}=0.5, \mathrm{~m}=0.5, \mathrm{Gr}=5, \operatorname{Pr}=2$ and $T=2$.

The effect of the Hall parameter $m$ on the transient axial velocity and transverse velocity is shown in Figs 3 and 4 respectively for the rotation parameter $\Omega=0.1$, Grashof number $G r=5$, Prandtl number $\operatorname{Pr}=2$, Hartmann number $\mathrm{M}=0.5$ and time $T=2$. It is observed that due to an increase in the Hall parameter, there is an increase in both the axial and transverse velocity components.

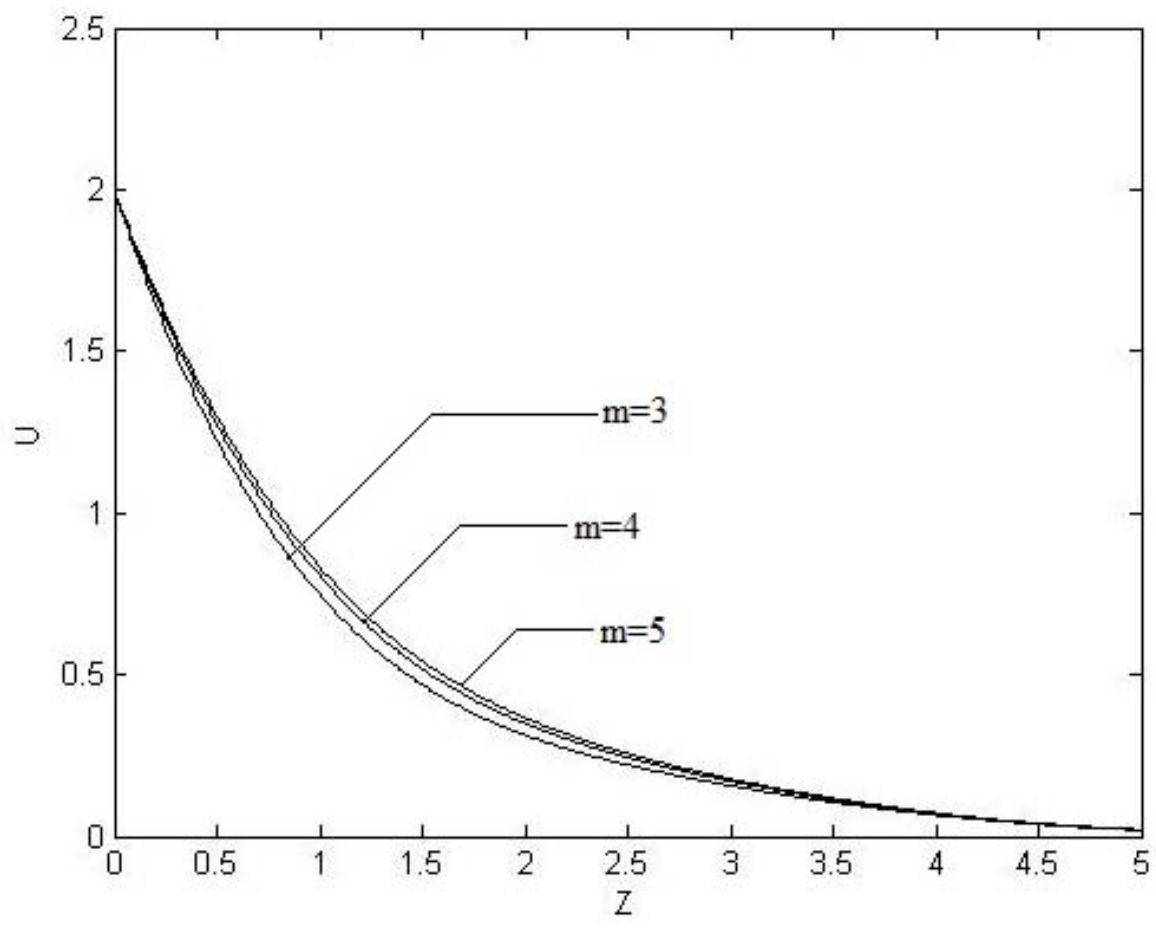

Fig.3. Axial velocity profile for $\Omega=0.1, \mathrm{M}=0.5, \mathrm{Gr}=5, \mathrm{Pr}=2$ and $T=2$. 


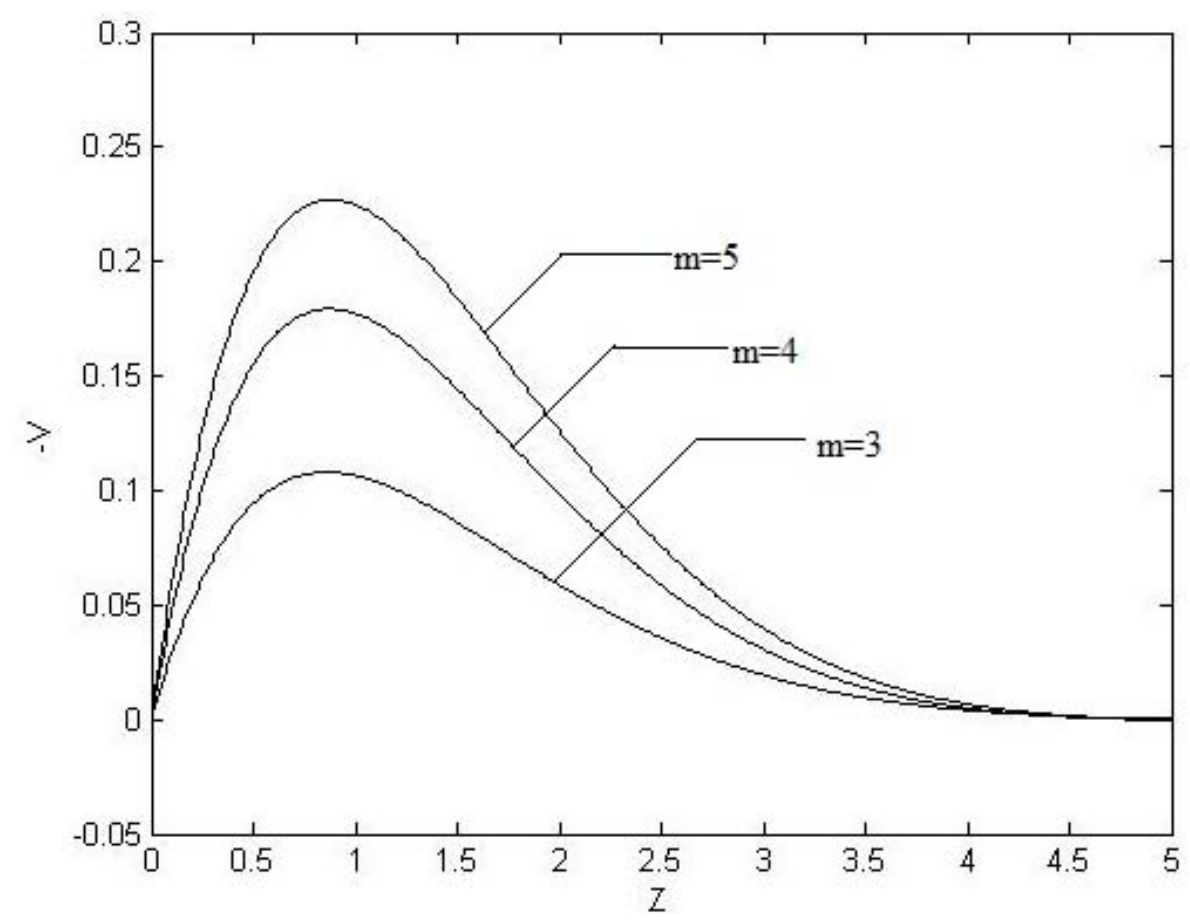

Fig.4. Transverse velocity profile for $\Omega=0.1, \mathrm{M}=0.5, \mathrm{Gr}=5, \operatorname{Pr}=2$ and $T=2$.

The effect of the magnetic field parameter on the transient axial velocity and transverse velocity is shown in Figs 5 and 6 respectively for the rotation parameter $\Omega=0.1$, Grashof number $\mathrm{Gr}=5$, Prandtl number $\operatorname{Pr}=2$, Hall parameter $\mathrm{m}=0.5$, and time $T=2$. It is observed that with the increase in the Hartmann number the axial velocity component increases in the negative direction, and the transverse velocity component increases drastically with the increase in the Hartmann number but in the opposite direction.

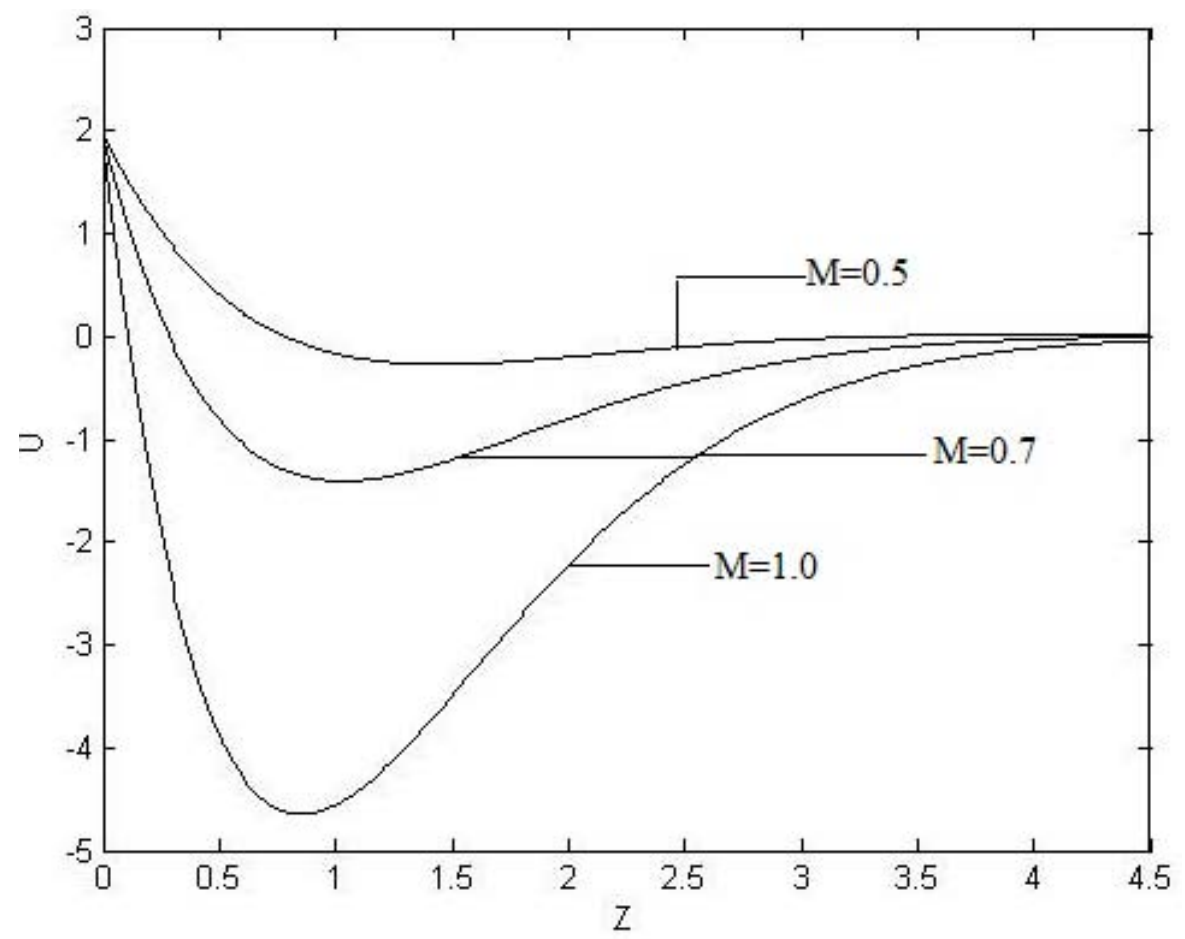

Fig.5. Axial velocity profile for $\Omega=0.1, \mathrm{~m}=0.5, \mathrm{Gr}=5, \operatorname{Pr}=2$ and $T=2$. 


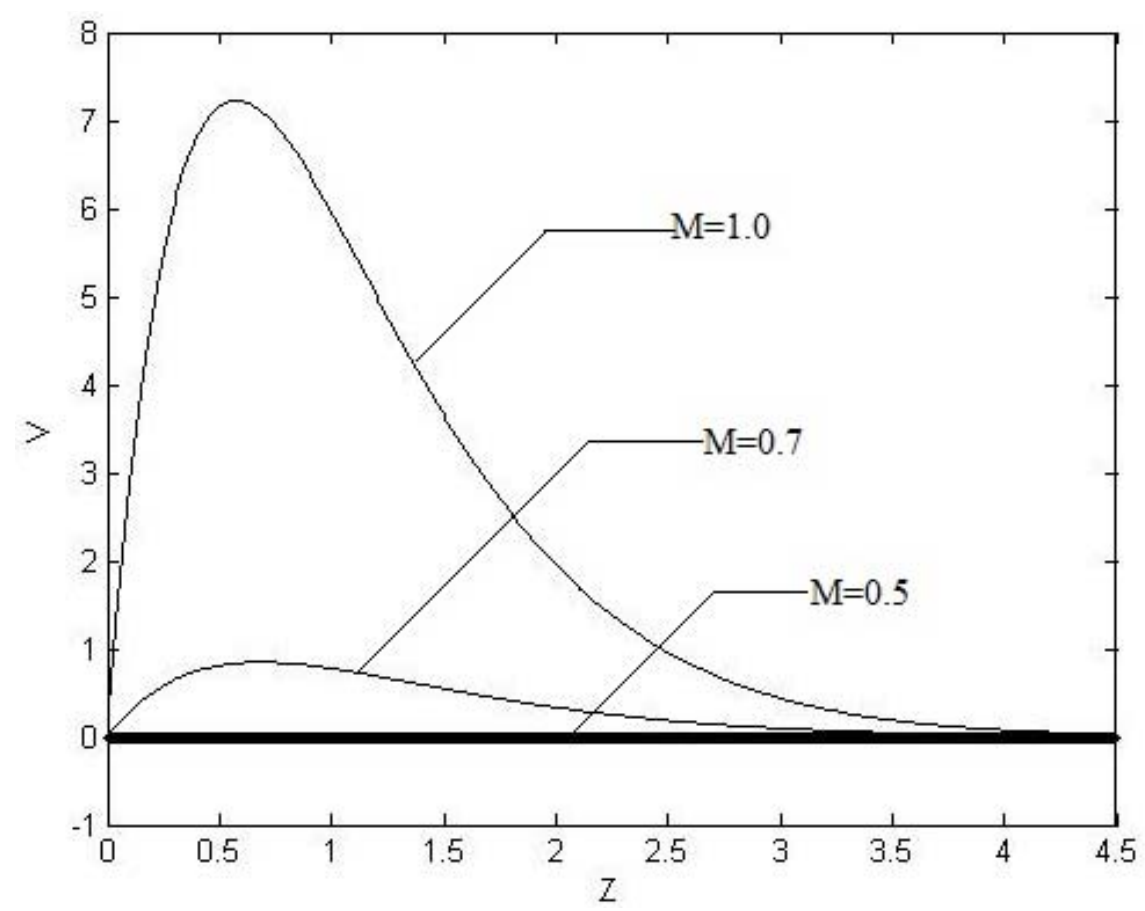

Fig.6. Transverse velocity profile for $\Omega=0.1, \mathrm{M}=0.5, \mathrm{Gr}=5, \operatorname{Pr}=2$ and $T=2$.

The effect of the Grashof number $\mathrm{G}$ on the transient axial velocity and transverse velocity is shown in Figs 7 and 8 respectively for the rotation parameter $\Omega=0.3$, Prandtl number $\operatorname{Pr}=2$, Hall parameter $\mathrm{m}=0.5$, Hartmann number $\mathrm{M}=0.5$ and time $T=2$. For $\Omega=0.1$, the transverse velocity component vanishes and thus the Grashof number has no effect on the transverse velocity in this case. From the graph it is observed that the axial velocity component decreases with the increase in the Grashof number and the transient velocity component increases with the increases in the Grashof number.

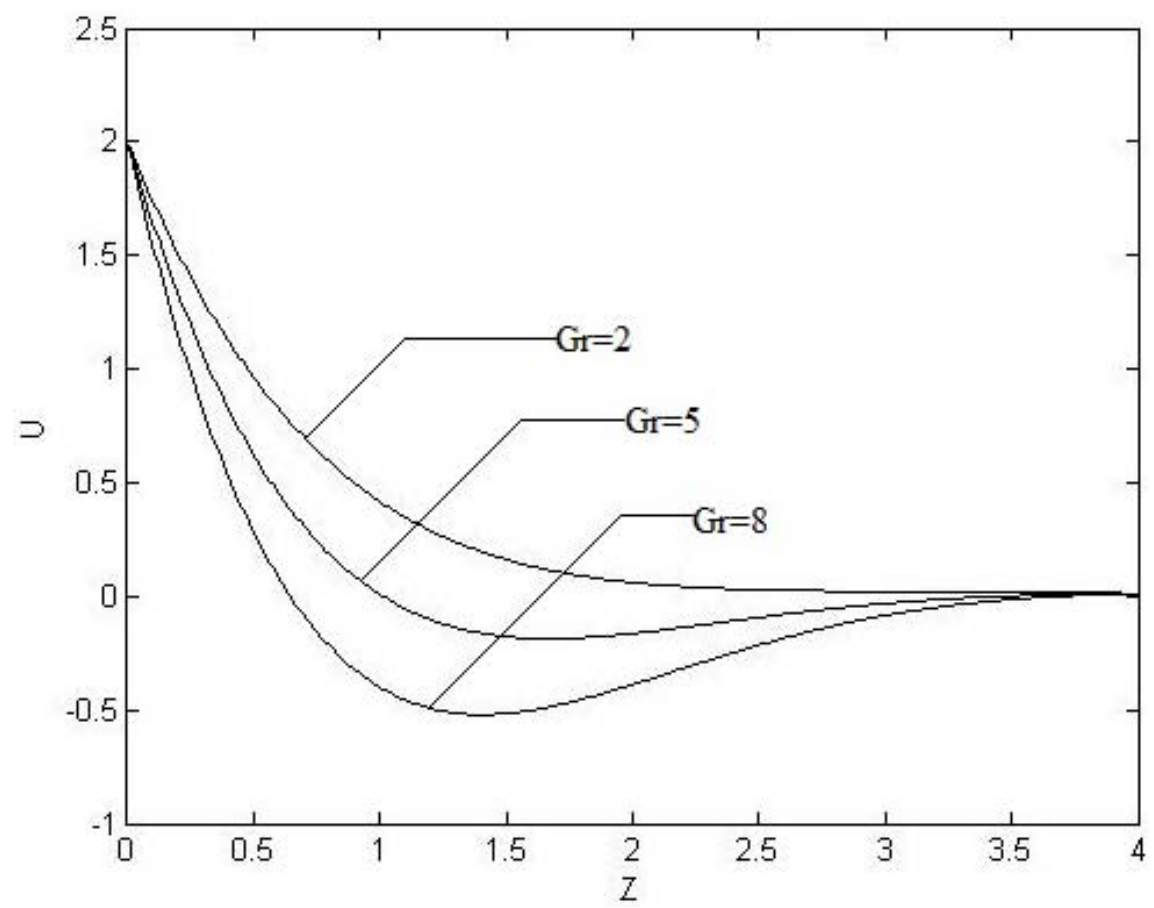

Fig.7. Axial velocity profile for $\Omega=0.3, \mathrm{M}=0.5, \mathrm{~m}=0.5, \operatorname{Pr}=2$ and $T=2$. 


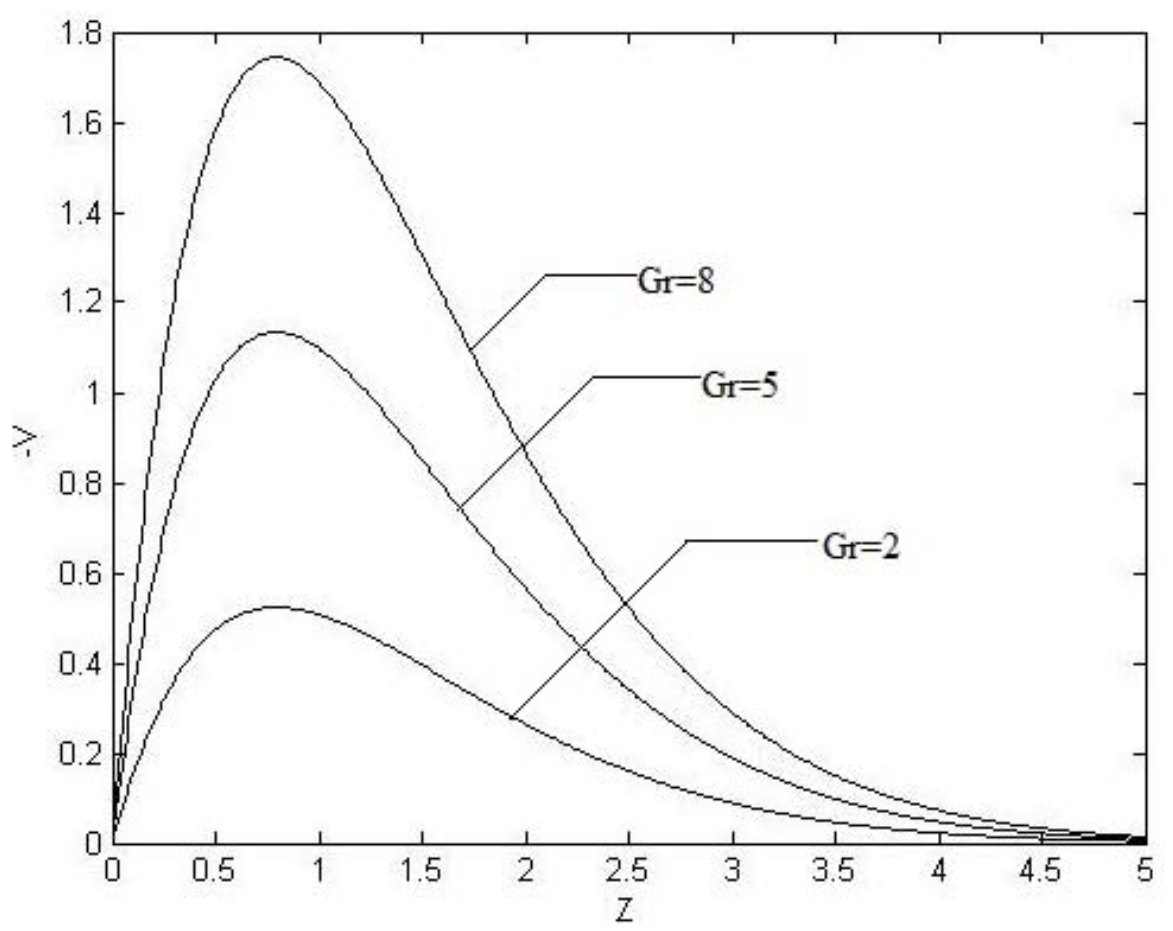

Fig.8. Transverse velocity profile for $\Omega=0.3, \mathrm{M}=0.5, \mathrm{~m}=0.5, \operatorname{Pr}=2$ and $T=2$.

The effect of the Prandtl number on the transient axial velocity and transverse velocity is shown in Figs 9 and 10 respectively, for the rotation parameter $\Omega=0.3$, Grashof's number $\mathrm{Gr}=5$, Hall parameter $\mathrm{m}=0.5$, Hartmann number $\mathrm{M}=0.5$ and time $T=2$. It is observed that the axial velocity component increases with the increase in the Prandtl number. However, the transient velocity component decreases.

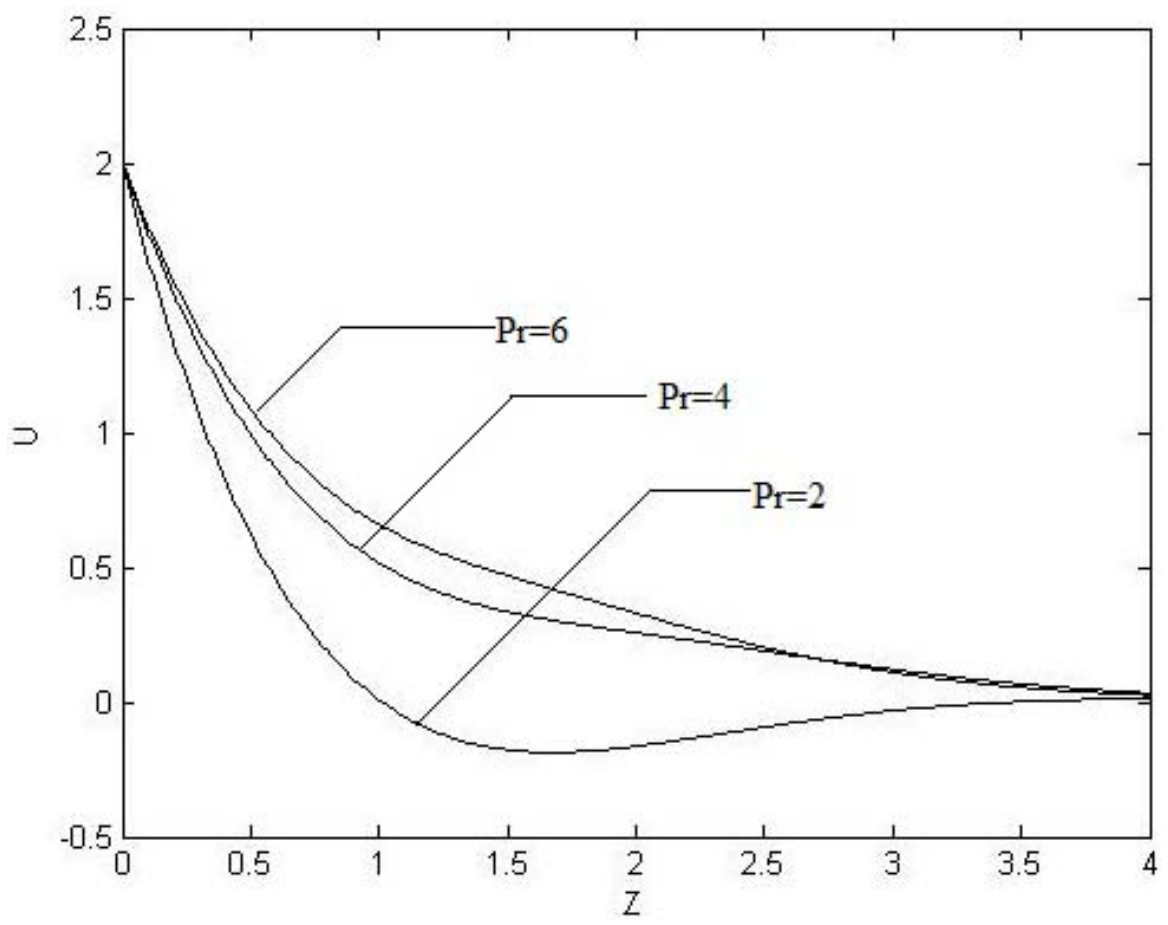

Fig.9. Axial velocity profile for $\Omega=0.3, \mathrm{~m}=0.5, \mathrm{G}=5$ and $T=2$. 


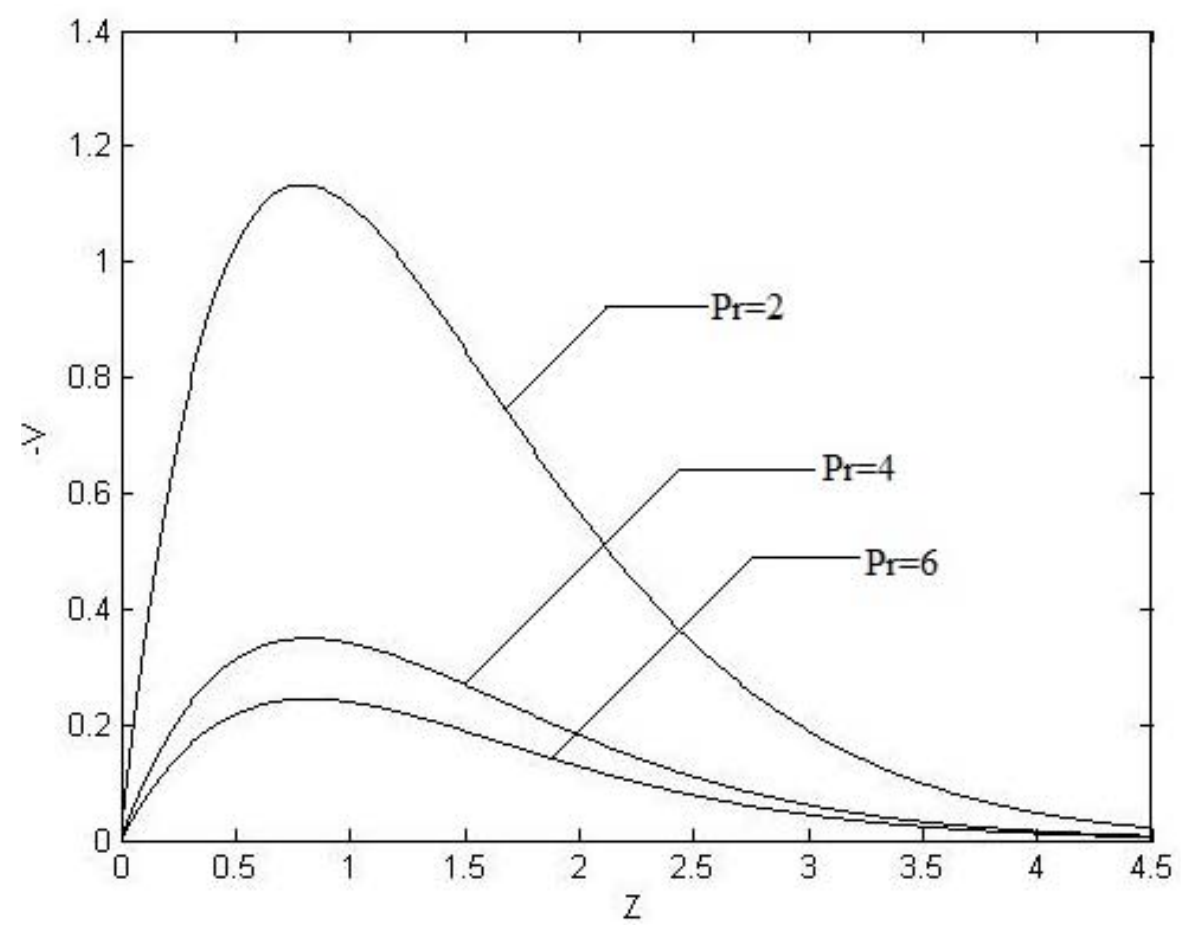

Fig.10. Axial velocity profile for $\Omega=0.3, \mathrm{M}=0.5, \mathrm{~m}=0.5, \mathrm{Gr}=5$ and $T=2$.

\section{Conclusion}

The simultaneous effects of Hall current and rotation on the MHD flow past an accelerated horizontal plate relative to a rotating fluid, with heat transfer characteristics have been studied. The effects of the Hall parameter, Hartmann number, rotation parameter, Grashof number and Prandtl number on the transient axial velocity and transverse velocity are presented graphically. It is also found that when the rotation parameter equals the value $M^{2} m /\left(1+m^{2}\right)$, for the given Hartmann number M and Hall parameter $\mathrm{m}$, the transverse component of velocity $V=0$ everywhere in the flow field so that the fluid moves in the direction of the plate only, which is the case in the absence of heat transfer also (Deka, 2008). It is also observed that, with the increase in the Hartmann number, the axial and transverse velocity increases in an opposite direction to that obtained by increasing the Hall parameter and rotation parameter.

\section{Nomenclature}

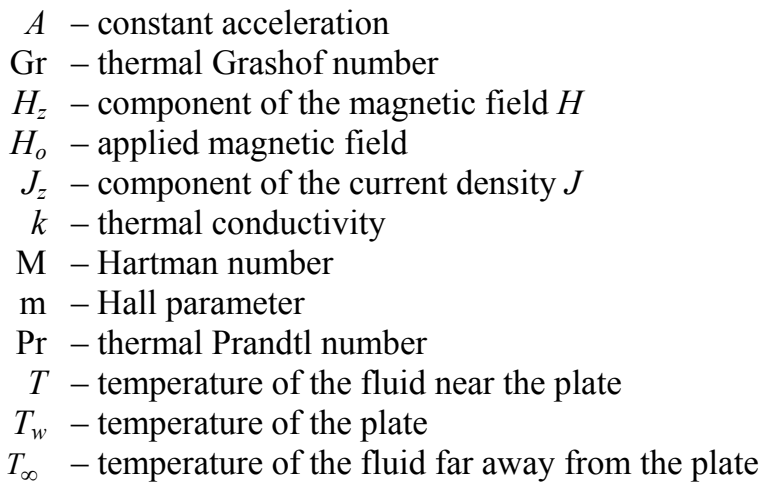




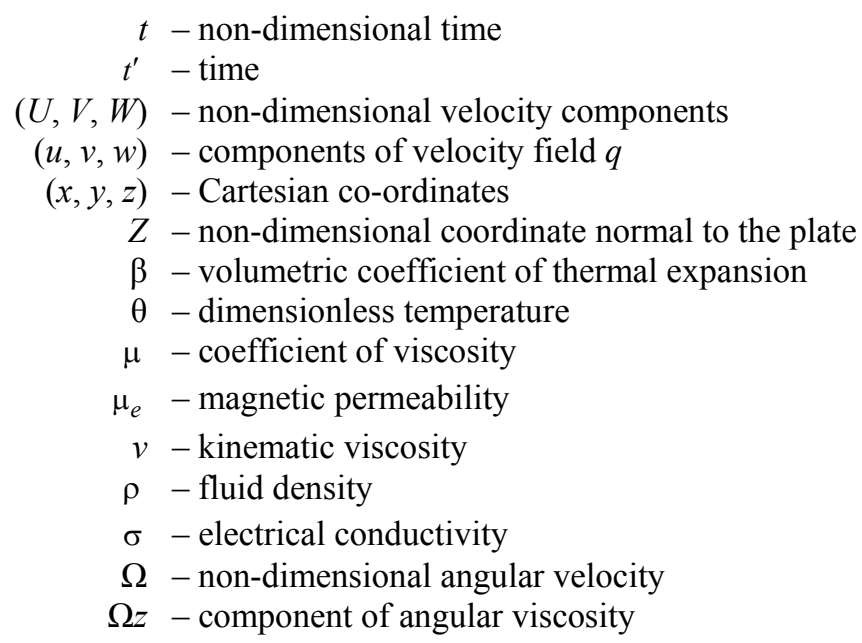

\section{References}

Abdul Maleque Kh. and Abdus Sattar (2005): The effects of variable properties and Hall current on steady MHD laminar convective fluid flow due to a porous rotating disk. - International Journal of Heat and Mass Transfer, vol.48, pp.4963-4972.

Abramowitz M. and Stegun I.A. (1965): Handbook of Mathematical Functions. - New York: Dover Publication.

Barik R.N., Dash G.C. and Rath P.K. (2013): Hall effects on unsteady MHD flow between two rotating disc with non coincident parallel axes. - Prec. Natl. Acad. Sci. India, Sect. A Phys. Sci. Vol.83, No.1, pp.21-27.

Barali A. and Borkakati A.K. (1982): The effect of Hall current on MHD flow and heat transfer between two parallel porous plates. - Applied Scientific Research, vol.39, pp.155-165.

Chauhan D.S. and Rastogi P. (2012): Heat transfer effects on rotating MHD Couette flow in a channel partially filled by a porous medium with hall current. - Journal of Applied Science and Engineering, vol.15, No.3, pp.281-290.

Cowling T.G. (1957): Magnetohydrodynamics. - p.101. Interscience, New York.

Deka R.K. (2008): Hall effects on MHD flow past an accelerated plate. - Theoret. Appl. Mech., vol.35, No.4, pp.333346.

Ghosh S.K., Bég O. Anwar and Narahari M. (2009): Hall effects on MHD flow in a rotating system with heat transfer characteristics. - Meccanica, vol.44, pp.741-765.

Hetnarski R.B. (1975): An algorithm for generating some inverse Laplace transforms of exponential form. - ZAMP 26, pp.249-253.

Pop I. (1971): The effect of Hall currents on hydromagnetic flow near an accelerated plate. - J. Math. Phys. Sci., vol.5, pp.375-379.

Rossow V.J. (1960): On Rayleigh's problem in magnetohydrodynamics. - Physics of Fluids, vol.3, No.3, pp.395398.

Singh A.K. (1984): Hall effects on MHD free-convection flow past an accelerated vertical porous plate. - Astrophysics and Space Science, vol.102, pp.213-221.

Sulieman H. and Qatanani N.A. (2012): Hydrodynamic Rayleigh problem with Hall effect. - IJMER, vol.2, No.1, pp.390-402. 